\title{
Phylogenetic diversity of acidophilic actinomycetes from Malaysia
}

\author{
Hideyuki Muramatsu1, Ryuji Murakami², Zool Hilmi Ibrahim ${ }^{3}$, Kana Murakami², Neelam Shahab ${ }^{3}$ \\ and Koji Nagai ${ }^{1}$
}

The Journal of Antibiotics (2011) 64, 621-624; doi:10.1038/ja.2011.57; published online 27 July 2011

Keywords: acidophilic actinomycetes; Malaysian actinomycetes; phylogenetic diversity

Actinomycetes produce various physiologically active compounds, including antibiotics. Although the discovery of new active compounds has slowed recently, some novel compounds from nonsoil borne actinomycetes; for example, marine actinomycetes, have been reported. ${ }^{1}$ Thus, discoveries of new taxa will expand the diversity of resources available for screening programs. Basilio et al. ${ }^{2}$ reported that actinomycetes isolated in alternative selections of $\mathrm{pH}$ and salinity present a significant capacity to produce compounds with antimicrobial activity. Like other actinomycetes, marine actinomycetes and other rarely targeted strains, for example, acidophilic actinomycetes, are expected to produce a diverse array of active compounds.

Research on acidophilic actinomycetes has been carried out since the 1960s. ${ }^{3,4}$ Recently, several new taxa of acidophilic actinomycetes have been described. In 2003, Kim et al. ${ }^{5}$ described the genus Streptacidiphilus belonging to the family Streptomycetaceae. The genera Actinospica and Catenulispora were described in 2006 by Cavaletti et al. ${ }^{6}$ and Busti et al., ${ }^{7}$ respectively. At the same time, the suborder Catenulisporineae, which includes Actinospica and Catenulispora but no neutrophilic genera, was described. ${ }^{6}$ Some species of Streptomyces were also described as acidophilus. ${ }^{8,9}$

The soil of Malaysia is classified as latosol, which has a low $\mathrm{pH}$. Zaidey et al. ${ }^{10}$ characterized soil properties of forests in Peninsular Malaysia, and reported $\mathrm{pH}$ values ranging from 4.15 to 5.89 . Williams et al. ${ }^{4}$ suggested that acidophilic actinomycetes are widely distributed in acidic soils. Some of the new acidophilic taxa mentioned above were isolated from acidic soils. ${ }^{8,9}$ Therefore, Malaysian soils and related materials are expected to be good sources of acidophilic bacteria, and an inventory of acidophilic actinomycetes from Malaysia would be useful for further studies in this field of research.

To expand the diversity of the microbial library for natural productbased drug discovery programs, we isolated acidophilic actinomycetes from soil and leaf litter samples collected from Malaysian forests. Here, we report on the novelty and phylogenetic diversity of these isolates.
Soil and leaf litter samples were collected from several forests in Malaysia in 2003. The forests were located at Mersing in the state of Johore, Mata Air in Perlis, Jerantut in Pahang and Endau Rompin National Park in Pahang. Acidophilic actinomycetes were isolated by the dilution-plating method on acidic humic acid-vitamin agar, which is modified humic acid-vitamin agar $^{11}$ adjusted to $\mathrm{pH} 4.0$ with $\mathrm{H}_{2} \mathrm{SO}_{4}$ and supplemented with kabicidin $\left(0.125 \mathrm{mg} \mathrm{ml}^{-1}\right.$, Wako Pure Chemical Industries, Osaka, Japan). Before inoculation, soil or leaf litter samples were suspended in $80 \%$ Percoll (GE Healthcare, Tokyo, Japan), and after centrifugation $(1000 \times g, 10 \mathrm{~min})$ the supernatant was incubated with penicillin $G\left(0.2 \mathrm{mg} \mathrm{ml}^{-1}\right)$ in neutral conditions for $4 \mathrm{~h}$. Neutrophilic fast-growing bacteria, including actinomycetes, decreased to approximately $1 \%$ of their original population size during this incubation (data not shown). The incubated supernatant was serially diluted and inoculated onto acidic humic acid-vitamin agar plates. The isolation plates were incubated at $30{ }^{\circ} \mathrm{C}$ for $2-4$ weeks. The isolates were incubated on acidic and neutral-diluted yeast-starch agar plates containing $0.4 \mathrm{~g}$ yeast extract, $2 \mathrm{~g}$ soluble starch, and $18 \mathrm{~g}$ agar (separately autoclaved) in $1000 \mathrm{ml}$ tap water. The $\mathrm{pH}$ of each medium was adjusted with $\mathrm{H}_{2} \mathrm{SO}_{4}$ or $\mathrm{NaOH}$, and growth of isolates on media of different $\mathrm{pHs}$ was used to confirm their acidophilic properties. In some cases, the acidophilic properties were further confirmed on medium with $\mathrm{pH}$ adjusted using $5 \mathrm{~mm}$ phosphate buffer because the $\mathrm{pH}$ of the medium sometimes changed during bacterial growth.

For all isolates, partial 16S rDNA sequences (more than 500 bases in length) were determined by the method described previously. ${ }^{12}$ The medium for direct PCR was adjusted to $\mathrm{pH} 5.0$ with $\mathrm{H}_{2} \mathrm{SO}_{4}$. In cases where there were low similarity values to the closest known species, nearly complete sequences of $16 \mathrm{~S}$ rDNA of the isolates were determined as described previously. ${ }^{13}$

Operational taxonomic units (OTUs) were defined as groups with sequence similarity values of $98.7 \%$ or higher. This value of this criterion was based on the study of Stackebrandt and Ebers. ${ }^{14}$

${ }^{1}$ Fermentation Research Div., Astellas Research Technologies Co., Ltd., Ibaraki, Japan; ${ }^{2}$ Pharmacology Research Labs, Astellas Pharma Inc., Ibaraki, Japan and ${ }^{3}$ Industrial Biotechnology Research Centre, SIRIM Berhad, Selangor, Malaysia

Correspondence: H Muramatsu, Fermentation Research Division, Astellas Research Technologies Co., Ltd., 21 Miyukigaoka, Tsukuba-shi, Ibaraki 305-8585, Japan.

E-mail: hideyuki.muramatsu@jp.astellas.com

Received 14 January 2011; revised 24 May 2011; accepted 7 June 2011; published online 27 July 2011 
After definition of the OTUs, the genera of each OTU was estimated from BLAST search ${ }^{15}$ (http://blast.ddbj.nig.ac.jp/top-j.html) results and/or phylogenetic analyses. The phylogenetic trees were constructed using Clustal $\mathrm{X}^{16}$ by the neighbor-joining method, ${ }^{17}$ using nearly complete sequences of $16 \mathrm{~S}$ rDNA.

To evaluate the potency of antibiotics production by the isolates, their anti-Aspergillus niger (ATCC 9642) activities were measured using the agar plug assay on Sabouraud's dextrose agar. After overnight incubation at $30^{\circ} \mathrm{C}$, the agar plugs were removed and the inhibition zones were measured.

In this study, 122 actinomycetes were isolated in acidic conditions from 21 soil samples and 21 leaf litter samples collected from Malaysian forests. We isolated 43 isolates from soil samples and 79 isolates from leaf litter samples. The isolates were assigned to 33 OTUs on the basis of their partial 16S rDNA sequences (Table 1). The GenBank/EMBL/DDBJ accession numbers for the 16S rRNA gene sequences of representative isolates from each OTU are AB604839AB604871.

Sequence analyses revealed that the isolates/OTUs belonged to several genera as follows: Actinoallomurus, Actinospica, Kitasatospora,
Nocardia, Streptacidiphilus and Streptomyces. The one remaining OTU could not be assigned to any described genus. High phylogenetic diversity was observed not only at the inter-generic level but also at the intra-generic level as shown in Table 1.

Many OTUs (21/33) showed less than $98.7 \%$ similarity to the closest known species, suggesting that these are candidates for new species. Among the OTUs, 23 were confirmed as acidophilus in growth tests on buffer-containing media (Table 1). Among these 23 confirmed acidophilus OTUs, $82.6 \%(19 / 23)$ showed low similarity to their closest species.

All the isolates belonging to Actinospica, which is an acidophilic genus, were isolated from leaf litter samples. OTU-5, 6, 8 and 9 showed obligate acidophilic properties. The growth of OUT-7 on buffer-containing media has not been tested yet. In contrast with isolates belonging to Actinospica, most of the isolates belonging to Streptacidiphilus, which is also an acidophilic genus, were isolated from soil samples (22/25). These OTUs showed moderate acidophilic properties. One OTU, OTU-31, contained isolates with neutrotolerant properties. The isolates belonging to OTU-31 had various growth $\mathrm{pH}$ ranges; for example, the growth $\mathrm{pH}$ range of isolate 645259 was

Table 1 OTUs containing isolates obtained from soil and leaf litter samples from Malaysian forests

\begin{tabular}{|c|c|c|c|c|c|c|}
\hline OTU & Genus & $\begin{array}{l}\text { Closest species } \\
\text { (Accession number) }\end{array}$ & $\begin{array}{l}\text { Similarity } \\
\text { value (\%) }\end{array}$ & 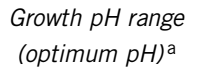 & Isolate count & $\begin{array}{l}\text { Isolates in } \\
\text { previous study }\end{array}$ \\
\hline OTU-1 & Actinoallomurus & A. purpureus (AB364588) & 98.35 & $5.0-6.0(6.0)$ & 1 & 0 \\
\hline OTU-2 & & A. spadix (AB364581) & 98.27 & $4.0-5.0(5.0)$ & 1 & 0 \\
\hline OTU-3 & & A. iriomotensis (AB364586) & 99.42 & $5.0-6.0(6.0)$ & 5 & 8 \\
\hline OTU-4 & & A. spadix (AB364581) & 98.83 & $5.0-6.0(5.0)$ & 2 & 0 \\
\hline OTU-5 & Actinospica & A. robiniae (AJ865863) & 97.72 & $5.0(5.0)$ & 3 & 0 \\
\hline OTU-6 & & A. acidiphila (AJ865861) & 98.69 & $4.0-5.0(5.0)$ & 25 & 0 \\
\hline OTU-7 & & A. robiniae (AJ865863) & 99.44 & nt & 1 & 0 \\
\hline OTU-8 & & A. acidiphila (AJ865861) & 99.04 & $4.0-5.0(5.0)$ & 1 & 0 \\
\hline OTU-9 & & A. acidiphila (AJ865861) & 99.03 & $5.0(5.0)$ & 2 & 0 \\
\hline OTU-10 & Streptomyces & S. mobaraensis (DQ442528) & 98.32 & $4.0-6.0(4.0)$ & 1 & 0 \\
\hline OTU-11 & & S. griseocarneus (X99943) & 96.36 & $4.0-6.0(5.0)$ & 5 & 0 \\
\hline OTU-12 & & S. ferralitis (AY262826) & 98.13 & $4.0-6.0(5.0)$ & 4 & 0 \\
\hline OTU-13 & & S. hebeiensis (AB249956) & 98.31 & $4.0-6.0(5.0)$ & 5 & 0 \\
\hline OTU-14 & & S. aureofaciens (Y15504) & 98.68 & $4.0-6.0(5.0)$ & 15 & 5 \\
\hline OTU-15 & & S. paucisporeus (AY442267) & 98.23 & $4.0-6.0(5.0)$ & 1 & 0 \\
\hline OTU-16 & & S. hebeiensis (AB249956) & 98.22 & $4.0-6.0(5.0)$ & 1 & 0 \\
\hline OTU-17 & & S. spinoverrucosus (AB184578) & 98.23 & $4.0-6.0(6.0)^{c}$ & 2 & 0 \\
\hline OTU-18 & & S. ferralitis (AY262826) & 98.49 & $4.0-8.0(7.0)$ & 2 & 0 \\
\hline OTU-19 & & S. parvisporogenes (AY999865) & 98.27 & $6.0-7.0(7.0)$ & 2 & 0 \\
\hline OTU-20 & & S. seoulensis (AB249970) & 99.62 & $4.0-7.0(6.0)$ & 4 & 88 \\
\hline OTU-21 & & S. coerulescens (AB184122) & 99.23 & $5.0-8.0(7.0)$ & 1 & 1 \\
\hline OTU-22 & & S. griseochromogenes (AJ310923) & 99.41 & $5.0-8.0(7.0)$ & 1 & 55 \\
\hline OTU-23 & & S. kagawaensis (AB184496) & 100 & nt & 2 & 54 \\
\hline OTU-24 & & S. misionensis (EF178678) & 100 & nt & 1 & 12 \\
\hline OTU-25 & & S. griseoplanus (AB184138) & 99.03 & nt & 1 & 3 \\
\hline OTU-26 & & S. prunicolor (DQ026659) & 99.44 & nt & 2 & 0 \\
\hline OTU-27 & Kitasatospora & K. sampliensis (AY260167) & 98.12 & $4.0-6.0(5.0)$ & 4 & 0 \\
\hline OTU-28 & Streptacidiphilus & S. oryzae (DQ208700) & 97.63 & $4.0-6.0(5.0)$ & 5 & 0 \\
\hline OTU-29 & & S. jiangxiensis (AB249948) & 98.49 & $4.0-6.0(5.0)$ & 8 & 0 \\
\hline OTU-30 & & S. neutrinimicus (AB249949) & 98.66 & $4.0-6.0(4.0)$ & 4 & 0 \\
\hline OTU-31 & & S. neutrinimicus (AB249949) & 98.46 & $4.0-7.0(5.0)^{d}$ & 8 & 5 \\
\hline OTU-32 & Nocardia & N. miyunensis (GQ376179) & 98.66 & $4.0-6.0(6.0)$ & 1 & 0 \\
\hline OTU-33 & - & Streptoalloteichus hindustanus (D85497) & 91.04 & $4.0-5.0(4.0)$ & 1 & 0 \\
\hline
\end{tabular}

Abbreviations: nt, not tested; OTUs, operational taxonomic units.

aGrowth $\mathrm{pH}$ range was determined on media adjusted to $\mathrm{pH} 3.0,4.0,5.0,6.0,7.0$ and 8.0, containing 5 mm phosphate buffer.

b Isolates previously isolated in neutral conditions from Malaysian soil samples ${ }^{12}$ (isolate count).

cTrace growth on $\mathrm{pH}$ 7.0.

'Trace growth on
dNeutrotolerant. 


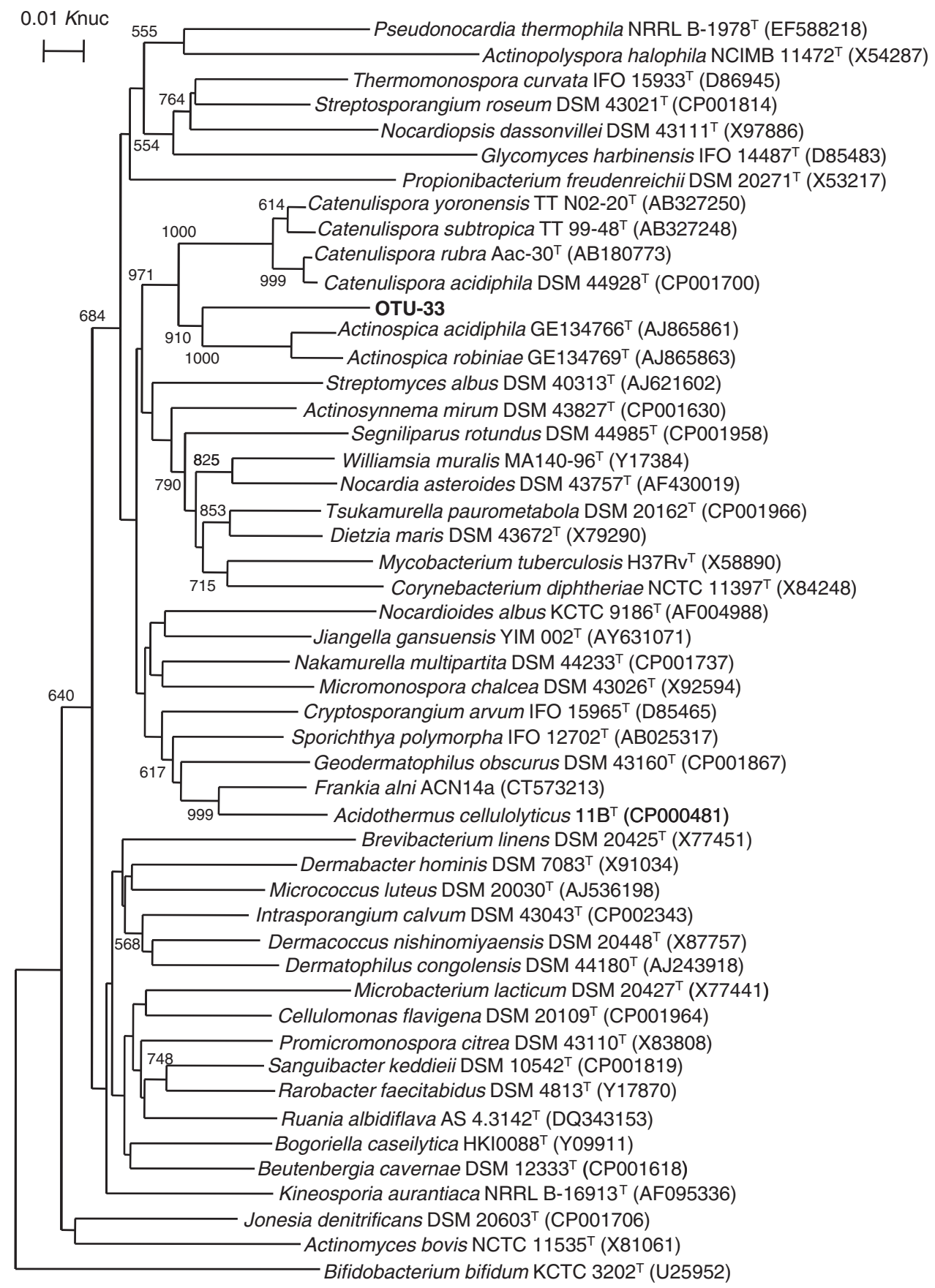

Figure 1 Phylogenetic tree of OTU-33 based on nearly complete 16S rDNA sequences. Tree contains members from all Actinomycetales families and all type species of the suborder Catenulispolineae. Numbers on branches are confidence limits (estimated from bootstrap analysis with 1000 replicates). Values greater than 500 are shown.

5.0-7.0 (optimum; 6.0), while that for isolate 645278 was $4.0-6.0$ (optimum; 5.0). The genus Actinoallomurus belonging to the family Thermomonosporaceae was described by Tamura et al. ${ }^{18}$ in 2009. Most of the isolates belonging to Actinoallomurus were isolated from leaf litter samples. OTU-3 was closely related to Actinoallomurus iriomotensis. The growth $\mathrm{pH}$ range of $A$. iriomotensis is $5.0-6.0,{ }^{18}$ consistent with the acidophilic property of OTU-3. The OTUs belonging to Streptomyces could be classified into three groups; A, B and C. Group A consisted of eight OTUs, OTU-10 to 17, which showed acidophilic properties on buffer-containing media (Table 1). Group B consisted of five OTUs, OTU-18 to 22, which did not show acidophilic properties on buffer-containing media (Table 1), and therefore, are not acidophilus. Group C consisted of four OTUs, OTU-23 to 26. The growth of OTU-23 to 26 on buffer-containing media has not been tested, and their acidophilic properties remain unclear. Except for OTU-10 and 17, the OTUs belonging to group A were isolated from leaf litter samples. Most of these OTUs were obligate acidophilus. Three of the OTUs in Group B were closely related to known Streptomyces species. The isolates in this group were isolated from soil or leaf litter. All of the OTUs in group C were closely related to known Streptomyces 
species. Isolates in Group C tended to be isolated from soil. Although no obligate acidophilic Streptomyces species have been described, we obtained seven Streptomyces OTUs showing obligate acidophilus properties. Six of these were isolated mainly from leaf litter, and all showed low similarity values to known species. This result indicated that obligate acidophilic Streptomyces strains are widely distributed in the leaf litter environment, and have not been thoroughly investigated yet. Xu et al. ${ }^{9}$ described a subclade of neutrotolerant acidophilic Streptomyces based on their 16S rRNA gene sequences. The closest species to OTU-15 was Streptomyces paucisporeus (98.2\% similarity), which is a neutrotolerant acidophilus, while the other OTUs were not related to this subclade. Obligate acidophilic Streptomyces isolates do not form single cluster (data not shown). OTU-27 and OTU-32 belonged to Kitasatospora and Nocardia. All isolates belonging to OTU-27 were isolated from leaf litter. The isolate belonging to OTU-32 was isolated from soil.

The taxonomic novelty of OTU-33 was confirmed by BLAST search results and phylogenetic analysis. According to the BLAST search results, the OTU has relatively low similarity values (90.1-93.1\%, based on sequences longer than 1200 bases) to various species belonging to the suborders Jiangellineae, Pseudonocardineae, Micromonosporineae, Micrococcineae, Glycomycineae or Corynebacterineae. The phylogenetic tree constructed with members from all Actinomycetales families indicated that the OTU belongs to the suborder Catenulisporineae, which consists of the families Catenulisporaceae and Actinospicaceae (Figure 1). All members of the suborder are acidophilus. ${ }^{6,7,19,20}$ However, OTU-33 did not cluster with members of Actinospicaceae or members of Catenulisporaceae, indicating that it is a candidate for a new genus of a new family in the suborder Catenulisporineae.

In our previous study, ${ }^{12}$ we isolated actinomycetes from Malaysian soil in neutral conditions. In that study, we obtained partial $16 \mathrm{~s}$ rDNA sequences of 790 isolates. Of those 790 isolates, 231 could be assigned to the OTUs in this study. Most of them were assigned to the OTUs that did not show acidophilic growth on buffer-containing media (Streptomyces group B) or those that were not tested on buffercontaining media (Streptomyces group C). The remaining 18 isolates were assigned to acidophilic OTUs; OTU-3, -14 and -31 (Table 1). These could be explained by their neutrotolerant or weak acidophilic properties. As mentioned above, OTU-31 contained neutrotolerant isolates. This comparison strongly indicated that there is a little overlap between the series of acidophilic isolates and that of neutrally isolated strains.

To evaluate the potential of the acidophilic isolates for drug discovery screening programs, we examined their anti-Aspergillus niger activities. Out of 56 isolates, 35 (62.5\%) had anti-Aspergillus niger activity. This result is consistent with that reported by Basilio et al. ${ }^{2}$ Although the number of isolates in this study is too small for comparison with neutrophilic actinomycetes, these results indicated that acidophilic actinomycetes are useful as new sources of bioactive compounds for drug discovery.

In this study, we isolated actinomycetes assigned to 33 OTUs from soil and litter samples collected from Malaysian forests. Of the 33 OTUs, 23 were confirmed as acidophilus. These belonged to various taxa, six known genera and one undescribed genus. Of the 23 acidophilic OTUs, 19 showed low similarity values $(<98.7 \%)$ to the closest known species, indicating that they are candidates for new species. One of these OTUs is likely to be a member of a new family. There was little overlap between the acidophilic isolates and neutrally isolated strains. Many of the isolates showed anti-Aspergillus niger activities. These results indicate that acidophilic actinomycetes are a promising source of biological material for drug discovery screening programs.
Although these isolates have promising properties, some problems need to be resolved. The isolation of acidophilic actinomycetes is often difficult because of contamination by fungi. Although most fungi grow well in acidic conditions, most antifungal agents; for example, nystatin, are unstable in such conditions. During these investigations, the isolation plates were frequently overgrown by fungi (data not shown). Another problem is how to rapidly and accurately assess the ability of each isolate to produce secondary metabolites. In addition, we have little experience of culturing actinomycetes in acidic conditions. To effectively use acidophilic actinomycetes in drug discovery programs, further investigations are required to optimize isolation, cultivation and screening methods.

\section{ACKNOWLEDGEMENTS}

We thank Reiko Usukura, Takako Ichimura and Hiromi Nakajima for their technical assistance.

1 Fenical, W. \& Jensen, P. R. Developing a new resource for drug discovery: marine actinomycete bacteria. Nat. Chem. Biol. 2, 666-673 (2006).

2 Basilio, A. et al. Patterns of antimicrobial activities from soil actinomycetes isolated under different conditions of pH and salinity. J. Appl. Microbiol. 95, 814-823 (2003).

3 Corke, C. T. \& Chase, F. E. Comparative studies of actinomycete populations in acid podzolic and neutral mull forest soils. Proc. Soil Sci. Soc. Am. 28, 68-70 (1964).

4 Williams, S. T., Davies, F. L., Mayfield, C. I. \& Khan, M. R. Studies on the ecology of actinomycetes in soil II. The $\mathrm{pH}$ requirements of streptomycetes from two acid soils. Soil. Biol. Biochem. 3, 187-195 (1971).

5 Kim, S. B., Lonsdale, J., Seong, C. N. \& Goodfellow, M. Streptacidiphilus gen. nov., acidophilic actinomycetes with wall chemotype I and emendation of the family Streptomycetaceae (Waksman and Henrici 1943AL) emend. Rainy et al. 1997. Antonie van Leeuwenhoek. 83, 107-116 (2003).

6 Cavaletti, L. et al. Actinospica robiniae gen. nov., sp. nov. and Actinospica acidiphila sp. nov.: proposal for Actinospicaceae fam. nov. and Catenulisporineae subord. nov. in the order Actinomycetales. Int. J. Syst. Evol. Microbiol. 56, 1747-1753 (2006).

7 Busti, E. et al. Catenulispora acidiphila gen. nov., sp. nov., a novel, mycelium-forming actinomycete, and proposal of Catenulisporaceae fam. nov. Int. J. Syst. Evol. Microbiol. 56, 1741-1746 (2006).

8 Kim, S. B., Seong, C. N., Jeon, S. J., Bae, K. S. \& Goodfellow, M. Taxonomic study of neutrotolerant acidophilic actinomycetes isolated from soil and description of Streptomyces yeochonensis sp. nov. Int. J. Syst. Evol. Microbiol. 54, 211-214 (2004).

9 Xu, C. et al. Neutrotolerant acidophilic Streptomyces species isolated from acidic soils in China: Streptomyces guanduensis sp. nov., Streptomyces paucisporeus sp. nov., Streptomyces rubidus sp. nov. and Streptomyces yanglinensis sp. nov. Int. J. Syst. Evol. Microbiol. 56, 1109-1115 (2006)

10 Zaidey, A. K. et al. Characterizing soil properties of lowland and hill dipterocarp forests at Peninsular Malaysia. Int. J. Soil Sci. 5, 112-130 (2010).

11 Hayakawa, M. \& Nonomura, H. Humic acid-vitamin agar, a new medium for selective isolation of soil actinomycetes. J. Ferment. Technol. 65, 501-509 (1987).

12 Muramatsu, H., Shahab, N., Tsurumi, Y. \& Hino, M. A comparative study of Malaysian and Japanese actinomycetes using a simple identification method based on partial $16 \mathrm{~S}$ rDNA sequence. Actinomycetologica 17, 33-43 (2003).

13 Muramatsu, H., Mokhtar, S. I., Katsuoka, M. \& Ezaki, M. Phylogenetic analysis of immunosuppressant FK506-producing streptomycete strains. Actinomycetologica 19, 33-39 (2005).

14 Stackebrandt, E. \& Ebers, J. Taxonomic parameters revisited: tarnished gold standards. Microbiol. Today 33, 152-155 (2006).

15 Altschul, S. F. et al. Gapped BLAST and PSI-BLAST: a new generation of protein database search programs. Nucleic Acids Res. 25, 3389-3402 (1997).

16 Thompson, J., Gibson, T. J., Plewniak, F., Jeanmougin, F. \& Higgins, D. G. The Clustal X windows interface: flexible strategies for multiple sequence alignment aided by quality analysis tools. Nucleic Acids Res. 24, 4876-4882 (1997).

17 Saitou, N. \& Nei, M. The neighbor joining method: a new method of constructing phylogenetic trees. Mol. Biol. Evol. 6, 514-525 (1987).

18 Tamura, T., Ishida, Y., Nozawa, Y., Otoguro, M. \& Suzuki, K. Transfer of Actinomadura spadix Nonomura and Ohara 1971 to Actinoallomurus spadix gen. nov., comb. nov., and description of Actinoallomurus amamiensis sp. nov., Actinoallomurus caesius sp. nov., Actinoallomurus coprocola sp. nov., Actinoallomurus fulvus sp. nov., Actinoallomurus iriomotensis sp. nov., Actinoallomurus luridus sp. nov., Actinoallomurus purpureus sp. nov. and Actinoallomurus yoronensis sp. nov. Int. J. Syst. Evol. Microbiol. 59, 1867-1874 (2009)

19 Tamura, T., Ishida, Y., Sakane, T. \& Suzuki, K. Catenulispora rubra sp. nov., an acidophilic actinomycete isolated from forest soil. Int. J. Syst. Evol. Microbiol. 57, 2272-2274 (2007).

20 Tamura, T., Ishida, Y., Otoguro, M. \& Suzuki, K. Catenulispora subtropica sp. nov. and Catenulispora yoronensis sp. nov. Int. J. Syst. Evol. Microbiol. 58, 1552-1555 (2008). 